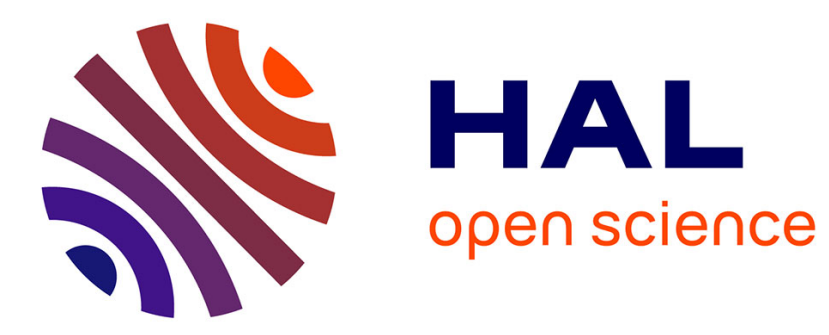

\title{
Le paradoxe du " langage commun" dans les entreprises : entre horizontalisation et contrôle social des pratiques langagières au travail \\ Vincent Mariscal
}

\section{- To cite this version:}

Vincent Mariscal. Le paradoxe du " langage commun " dans les entreprises: entre horizontalisation et contrôle social des pratiques langagières au travail. Langage et Société, 2016, pp.13-34. hal-03214671

\section{HAL Id: hal-03214671 \\ https://hal.science/hal-03214671}

Submitted on 3 May 2021

HAL is a multi-disciplinary open access archive for the deposit and dissemination of scientific research documents, whether they are published or not. The documents may come from teaching and research institutions in France or abroad, or from public or private research centers.
L'archive ouverte pluridisciplinaire HAL, est destinée au dépôt et à la diffusion de documents scientifiques de niveau recherche, publiés ou non, émanant des établissements d'enseignement et de recherche français ou étrangers, des laboratoires publics ou privés. 
Mariscal Vincent

Université catholique de Louvain, Institut Langage \& Communication, Laboratoire Valibel vincent.mariscal@yahoo.fr

\section{Le paradoxe du « langage commun » dans les entreprises : entre horizontalisation et contrôle social des pratiques langagières au travail}

Résumé : Cet article analyse une forme spécifique de contrôle du langage au travail apparue dans les années 1980, à travers l'étude de manuels de communication d'entreprise. Il s'agit de la notion de «langage commun », censée garantir la mobilisation de tous les acteurs en vue du succès de l'entreprise. Les auteurs des manuels envisagent les pratiques langagières au travail comme pouvant être parfaitement horizontales et symétriques. Le langage est, ainsi, considéré comme un « code », autant linguistique que socioculturel. En assurant son acquisition par tous les acteurs, on pourrait alors obtenir une intercompréhension parfaite. Nous défendons l'idée que cette vision repose sur le déni des rapports conflictuels inhérents à toute relation sociale, en particulier au travail, et qu'elle est basée sur une homogénéisation linguistique et culturelle particulièrement stricte, tout en mettant en scène une logique néolibérale, c'est-à-dire une société de libre-échange où les facteurs socioculturels et sociolinguistiques sont minorisés.

Abstract: Based on an examination of corporate communication manuals, this article analyses a specific type of language control in the workplace, 'common language', which emerged in the 1980s and was supposed to improve employee motivation and thus ensure a company's success. The authors of the manuals believe language practices in the workplace can be perfectly horizontal and symmetrical. Language is thus seen as a linguistic and sociocultural 'code'. If all players learn it, perfect mutual understanding can be achieved. We support the idea that this vision ignores the conflict inherent in all social relationships, particularly in the workplace, and that it is based on a particularly rigid linguistic and cultural homogeneity, while bringing into play a neoliberal logic; in other words, a free-exchange society where sociocultural and sociolinguistic factors are minoritised.

Mots clés : code, contrôle, horizontalisation, néolibéralisme, normalisation, prescription. 


\section{Introduction}

Cet article analyse une forme de contrôle social dont le langage au travail a fait l'objet à partir des années 1980. Il s'agit de la volonté, de la part du management, de mettre en place un «langage commun » au sein de l'entreprise. L'étude de ce phénomène organisationnel a été réalisée à partir d'un corpus composé de onze manuels de communication d'entreprise. Ce travail résulte du recensement des manuels de communication d'entreprise publiés en français depuis les années 1980, réalisé pour un projet de recherche mené dans le cadre d'une thèse (Mariscal 2015). Afin de sélectionner les ouvrages servant de base à notre corpus, nous nous sommes efforcés de sélectionner des auteurs présentant des approches différentes en matière de communication d'entreprise, de manière à ce que la vision que nous pourrions donner de cette littérature soit la plus proche possible des multiples axes coexistant dans ce domaine. Nous avons ensuite distribué ces manuels en deux groupes. D'un côté, nous avons des manuels traitant de la communication dite «interne », parfois nommée «marketing interne » [Béal, Frommer et Lestocart 2003, D’Almeida et Libaert 2010, Décaudin, Igalens et Waller 2006, Détrie et Meslin-Broyez 2001, Donjean 2007, Morel 2009]. Ces ouvrages ont pour but de théoriser et/ou d'enseigner des techniques employées par le management pour diriger des individus ou des groupes à l'intérieur d'une entreprise par l'intermédiaire de la communication sous différentes formes (pratiques langagières, journaux d'entreprise, Intranet, etc.). De l'autre côté, nous avons un certain nombre de manuels en lien avec les performances individuelles en termes de communication, destinés aux personnes souhaitant améliorer leurs performances communicationnelles, relationnelles et leur assertivité au travail [Cayatte 2008, Guilbert 2007, Lafrance et Lambotte 2008, Nahon et Taskin 2009, Sananès 2011]. Il faut insister sur le fait que ces deux types de manuels ne sont pas exclusifs car on peut retrouver des questions concernant la communication interne dans la seconde catégorie et vice-versa. Ces différents manuels ont d'ailleurs en commun l'objectif d'atteindre une certaine efficacité organisationnelle par la maîtrise individuelle et collective des pratiques langagières.

Nous avons recherché, dans ce corpus, notamment à l'aide du logiciel Lexico ${ }^{1}$, l'ensemble $^{\prime}$ des occurrences de «langage commun» afin d'observer précisément ce à quoi elles font référence dans les manuels étudiés. C'est ce qui nous a amenés à formuler notre hypothèse principale qui est qu'il existerait un paradoxe dans la conceptualisation de la notion de « langage commun » entre l'injonction à l'horizontalisation des échanges au travail et le fait que cet outil managérial soit envisagé comme une possibilité de contrôler socialement les pratiques langagières dans les entreprises. Nous avons également mené une recherche systématique des termes issus des sciences du langage que nous pourrions nous attendre à trouver dans des ouvrages tentant de théoriser les pratiques communicationnelles, comme « langage », « langagier(s), -ière(s) », « langue » et «parole ». D’une part, notre but est d'avoir une vision globale de la manière dont les pratiques langagières sont envisagées par les auteurs de ces ouvrages. D'autre part, l'absence de certains termes, le nombre réduit d'occurrences ou les définitions souvent élémentaires données nous ont permis de faire une seconde hypothèse de travail. Elle est que la réduction à l'essentiel des connaissances produites par les sciences du langage nous mène à penser que l'on veut ignorer l'hétérogénéité fondamentale des pratiques langagières et, ainsi, tenter d'écarter une grande part de l'imprévisibilité de cette activité humaine par excellence au travail (Boutet 2008), mais aussi d'euphémiser un rapport salarial intrinsèquement conflictuel (Lordon 2010).

Ce travail sociohistorique est pluridisciplinaire, il intègre des études venues d'horizons différents et, en premier lieu, les travaux en sociolinguistique et en analyse du discours de Josiane Boutet (2008) et de Bernard Gardin (2005) sur le langage au travail. Notre recherche s'inspire également de l'étude sociologique des idéaux-types circulant dans les manuels de

\footnotetext{
${ }^{1}$ Lexico3 est réalisé par le laboratoire SYLED-CLA ${ }^{2} T$, de l'Université Paris 3, Sorbonne Nouvelle.
} 
management menée par Luc Boltanski et Ève Chiapello (1999). Elle nous a aidés à étudier la manière dont les interactions langagières sont théorisées dans les manuels de communication d'entreprise et à analyser les arguments avancés par les auteurs, en lien avec la transformation du management à partir des années 1980, pour montrer la nécessité d'adopter un «langage commun » aujourd'hui en particulier dans les organisations mondialisées. Cet article se situant dans une perspective critique, nous verrons quels sont les problèmes posés par cette manière d'aborder le langage au travail. Ainsi, comme Boltanski et Chiapello (1999) l'ont fait pour les principales notions mobilisées dans le discours managérial contemporain, nous voulons comprendre comment la notion de «langage commun» a circulé et a évolué depuis la théorisation du management dans les années 1980. Nous avons également mobilisé des travaux issus de la sociologie critique du travail (par exemple Aubert et de Gaulejac 1991, Linhart 2015) et de la communication (par exemple Floris 1996, Mattelart 2011, Winkin 2001), de l'histoire des idées politiques depuis les années 1980 (Cusset 2008) et des réflexions sur le capitalisme néolibéral et sur l'horizontalisation des institutions à l'époque contemporaines (Lordon 2010, 2015).

Nous verrons, dans la première partie, la manière dont les théoriciens du management se saisissent, au début des années 1980, des enjeux stratégiques liés à la « libération » du langage au travail. Dans un deuxième temps, nous étudierons les différentes formes de «langage commun ». Cette seconde partie se compose de trois sous-sections. Dans la première, nous expliquerons de quelle manière la notion de «langage commun» est apparue dans les théorisations de la communication et du management. La seconde sous-section étudie l'idée de «culture commune », qui est la définition du «langage commun » la plus fréquemment rencontrée dans les manuels étudiés. Troisièmement, nous verrons que le «langage commun » est également défini comme étant un «code commun », code qui revêt des aspects autant linguistiques que socioculturels. Dans la dernière partie, nous montrerons que le «langage commun » correspond à une forme d'idéalisation des interactions langagières au travail intimement liée à l'idéologie néolibérale, censée masquer les aspects conflictuels inhérents au rapport salarial.

\section{Les enjeux stratégiques du langage au travail en France depuis les années 1980}

En France, au début des années 1980, la question du langage au travail a fait l'objet d'une certaine attention de la part du monde politique autant que de la part des théoriciens du management (Boutet 2008, Le Goff 1989). Émerge, à cette époque, un certain nombre de réflexions dont celle sur la nécessité de mettre en place une loi rendant l'expression des employés libre sur leur lieu de travail. Ces réflexions déboucheront sur la loi Auroux, votée en 1982 par le gouvernement Mauroy lors du premier mandat de François Mitterrand ${ }^{2}$ (cf. Boutet 2008, Gardin 2005 : 321-354, Le Goff 1989).

Ce moment de « libération » de la parole au travail correspond, de manière contingente, à l'apparition de la théorisation du management dit «participatif », provenant des États-Unis (voir par exemple Peters et Waterman 1983) et du Japon (voir par exemple Albert 1982, 1983, Archier 1981, Maury 1986, 1990), qui va cristalliser tous les espoirs d'une relance économique dans une France qui souffre des conséquences de la crise pétrolière de 1973. En effet, les modèles industriels américain et japonais connaissent alors un certain succès économique et ils étaient considérés comme exemplaires du soutien de la croissance par un nouveau modèle managérial. Dans ce modèle, la communication sous toutes ses formes, va prendre une place de premier plan (Mariscal 2015).

De cette manière, la libération de la parole au travail par force de loi et le projet néomanagérial se rencontrent et, durant les années 1980, les théoriciens du management

\footnotetext{
${ }^{2}$ Deuxième paragraphe de l'Art. L. 461-1. de la loi du 4 août 1982.
} 
semblent prendre véritablement la mesure des enjeux stratégiques liés à la parole au travail (Boutet 2008, Gardin 2005, Mariscal 2015). En effet, le management « participatif » va inspirer de nombreux théoriciens français, qui vont populariser l'idée selon laquelle la communication en général, et les pratiques langagières en particulier, devraient faire partie intégrante du processus productif de l'entreprise, c'est-à-dire contribuer, tout autant, à sa rentabilité, à sa compétitivité et au bien-être au travail (Floris 1996, Mariscal 2015).

Comme Luc Boltanski et Ève Chiapello (1999) l'ont montré cette évolution est, d'une certaine manière, l'aboutissement d'une demande sociale née de la critique, notamment syndicale, des organisations traditionnelles et, en particulier, de celle du paternalisme et du taylorisme. Dans ce sens, l'injonction à « communiquer» au travail peut être vue comme étant comparable à l'injonction à devenir «autonome », ces deux injonctions ayant fait l'objet, parallèlement, d'une gestion et d'une canalisation spécifiques par le management depuis une trentaine d'années (Boltanski et Chiapello 1999, Mariscal 2015). Ainsi, comme toute forme de libération impulsée, au départ, par le salariat, la libération du langage au travail s'est accompagnée de formes de normalisation permettant au management de continuer à maintenir une certaine efficacité productive.

C'est selon ce cheminement que le langage au travail est passé du statut d'excédent, non essentiel à la production, voire d'activité sanctionnée par les règlements internes des entreprises (Boutet 2008, Le Goff 1989), à celui de rouage essentiel du management, vision à laquelle la théorisation de la communication d'entreprise viendra contribuer à partir du milieu des années 1980. Il faut insister sur le fait que cette dernière n'est pas uniquement centrée sur le marketing ou la diffusion d'informations en interne. En effet, elle touche aux deux domaines essentiels du management que sont la mobilisation des salariés et le travail sur l'harmonisation des relations interpersonnelles au travail. Ainsi, alors même que la concurrence et l'individualisation sont de plus en plus fortes dans un contexte néolibéral et globalisé, il faudrait que chaque individu fasse en sorte, pour être et demeurer « employable », de rendre ses relations avec autrui efficaces, en d'autres termes, productives et non-conflictuelles (Mariscal 2015, 2016).

Il est notamment exigé des acteurs de l'entreprise, en particuliers des managers, qu'ils acquièrent une compétence spécifique : le sens de la «connectivité » (Boltanski et Chiapello 1999 : 151, 219). De cette manière, les managers sont appelés à améliorer la qualité de leurs relations avec leurs subordonnés, et à constituer et entretenir des « réseaux » professionnels nationaux et internationaux. Cette compétence va devenir une évidence qui, comme Nicole D'Almeida et Thierry Libaert $[2010: 29]^{3}$ le rappellent dans leur manuel de communication, s'est imposée de l'extérieur, par l'éclatement des organisations sur le plan «spatial», « temporel » et « statutaire ». Ce « monde connexionniste » (Boltanski et Chiapello 1999: 168, 217) est devenu un mode d'existence, une norme comportementale (Cusset $2008: 322-323$ ), et les réseaux sont considérés comme devant faire tenir ensemble les différents éléments d'une activité disséminée à travers le monde (Mattelart 2011 : 339, Castells 1998), de manière à ce que tous les acteurs composant l'organisation marchent à l'unisson (Lordon 2010).

Les réseaux devraient ainsi former une maille à travers laquelle l'entreprise est organisée de manière « horizontale » et « démocratique », par opposition à la « verticalité totalitaire » des modèles issus du XIX ${ }^{\text {ème }}$ siècle (Cusset 2008 : 322-323), en particulier du taylorisme. Les pratiques langagières sont appelées à consolider cette maille car, selon le manuel de Sébastien Nahon et Laurent Taskin [2009 : 100], « la source principale de la productivité réside dans le transfert des intentions, des actions et des savoirs des différents acteurs des processus de production ».

\footnotetext{
${ }^{3}$ Les références entre crochets correspondent aux termes, citations et notions tirés de notre corpus.
} 


\section{Les différentes formes de «langage commun »}

\subsection{L'horizontalisation de l'entreprise}

Nous allons débuter maintenant l'analyse proprement dite de notre corpus de manuels de communication d'entreprise. Le problème de l'asymétrie entre les acteurs de l'entreprise semble venir motiver une action directe du management et de la communication d'entreprise sur les pratiques langagières. Certains auteurs de manuels comme Jean-Pierre Béal, Franck Frommer et Pierre-André Lestocart [2003 : 134] notent que le top-management a tendance à s'exprimer «dans un langage technocratique et abstrait », ne permettant pas de donner accès aux informations qu'ils veulent transmettre à l'ensemble des acteurs se trouvant plus bas qu'eux dans la hiérarchie. Philippe Morel [2009 : 120] résume cette problématique dans son manuel en disant que «l'entreprise qui a décidé de parler doit veiller à être comprise par tous », car « chacun ne sera pas nécessairement familiarisé avec un discours de spécialiste ». La solution avancée est de faire un effort de vulgarisation en utilisant « un langage simple » et de proscrire " les tournures alambiquées, les figures de rhétorique et le jargon technique » [Cayatte 2008 : 88]. La réponse apportée à l'asymétrie est donc de l'ordre de la forme, de la stylistique à adopter pour permettre à tous les acteurs, ne disposant pas nécessairement des mêmes compétences linguistiques, d'avoir un accès efficace aux informations circulant dans l'entreprise, et surtout à celles provenant du top-management. En effet, les propos de la frange de la hiérarchie la plus qualifiée devraient être accessibles à la frange la moins qualifiée, supposée se trouver au bas de l'échelle hiérarchique. Cela reflète l'image classique d'une entreprise « verticale » où les compétences vont croissantes lorsque l'on se rapproche du haut de la pyramide hiérarchique, et où l'action de vulgarisation revient à « horizontaliser »son propos, c'est-à-dire à renverser le rapport de pouvoir classique existant entre les différentes classes socioprofessionnelles, tout du moins symboliquement.

Mais il ne s'agit pas d'adopter une posture égalitariste, c'est-à-dire de montrer une volonté d'abolir tout rapport de domination de classes, mais seulement d'éviter la «communication verticale » [Lafrance et Lambotte 2008 : 34] faisant obstacle à l'échange d'informations. En effet, pour que le langage émanant du top-management soit opérationnel et efficace, il faudrait que n'importe quel employé soit capable de comprendre rapidement « les tenants et aboutissants du message » [Lafrance et Lambotte 2008 : 34]. Donc, la communication verticale, réputée être celle de la hiérarchie traditionnelle, paternaliste et tayloriste est remise en cause et sert de base à un discours défendant l'horizontalité des rapports interpersonnels, mais en tant qu'objectif pratique et non pour défendre une quelconque idéologie sociale. Le but réel de cette horizontalisation semble être de favoriser la participation, au sens de l'implication, du partage de l'information et non du partage du pouvoir décisionnel (Mariscal 2015).

\subsection{Le « langage commun » comme culture d'entreprise}

C'est cela qui motive, dans les textes étudiés, l'intervention sur le langage au travail par la construction d'une communication que tous seraient capables d'interpréter. Cette communication est construite selon les prérogatives des instances managériales. De cette manière, le « cadre », qui désigne dans le manuel de André-A. Lafrance et François Lambotte [2008 : 33] la situation de communication, est appelé à être " adapté au contenu du message » et non l'inverse, ce qui est une indication du degré de normalisation envisagé par les auteurs. Par exemple, le lieu d'une réunion devra être adapté, par son environnement et par le profil ou le comportement des individus présents durant l'échange, au message que l'entreprise voudra faire passer, pour que celui-ci ne soit pas « pollué » et que l'on atteigne l'objectif visé [Lafrance et Lambotte 2008 : 33]. Ainsi, de manière contre-intuitive, c'est le contexte qui devrait être adapté au message et non le message au contexte. 
Pour ce faire, la communication d'entreprise préconise l'adoption d'un «langage commun $»^{4}$. Cette notion apparaît déjà chez les pionniers américains du nouveau management au début des années 1980, dans Le prix de l'excellence de Thomas Peters et Robert H. Waterman. Dans ce livre, les auteurs ont enquêté sur les entreprises ayant rencontré les meilleurs résultats aux États-Unis depuis le milieu des années 1970, et ils décrivent les méthodes de management de ces organisations comme étant celles dont on devrait s'inspirer pour atteindre le succès. Thomas Peters et Robert H. Waterman (1983 : 120) relient le « langage commun » aux propos du spécialiste en stratégie des organisations, Andrew Pettigrew, qui considère que le « façonnement de la culture » est le rôle du top-management et la clé du succès, comme le montreraient les expériences menées par des multinationales telles que HewlettPackard, IBM et Digital.

Le « langage commun » est ainsi relié à une notion fondamentale du nouveau management, qui est la «culture d'entreprise », cette dernière englobant, chez Thomas Peters et Robert H. Waterman (1983 : 120), le langage au même titre que les «symboles », les «idéologies », les «croyances », les « rituels » et les «mythes». Le langage est donc défini à partir de signifiants et d'archétypes socioculturels. Mais, l'argument avancé par Thomas Peters et Robert H. Waterman (1983) n'est pas de s'appuyer sur des bases socioculturelles existantes que l'on pense être partagées par le plus grand nombre dans une société donnée, mais de les créer de toute pièce, notamment en mettant en place des mots et des expressions spécifiques à l'entreprise afin de favoriser la cohésion entre les individus. Nous remarquons, ici, une véritable proximité entre le marketing qui est traditionnellement l'objet de la communication externe, et la communication d'entreprise, le «marketing interne » [cf. par exemple Béal, Frommer et Lestocart 2003]. Ainsi, Thomas Peters et Robert H. Waterman (1983 : 122) appellent de leurs vœux la mise en place d'un «nouveau langage », de «nouvelles métaphores et de nouveaux modèles » pour construire « un ensemble sensé, cohérent et mémorisable ». Nous voyons donc apparaître, chez ces pionniers du management contemporain, non pas des propositions concrètes pour mettre en place un « langage commun », mais un horizon idéal, esprit que nous retrouvons dans les manuels de communication d'entreprise, comme nous le verrons plus bas.

Nous trouvons en France, dès 1981, des propositions similaires chez les théoriciens du management. Par exemple, Georges Archier (1981 : 84), veut inciter les organisations, en s'inspirant du succès des entreprises japonaises, à créer un ensemble de pratiques culturelles et professionnelles communes médiées par le langage. Hervé Sérieyx (1982 : 56) fait de même en 1982, en appelant à la création d'un « langage commun à tous les niveaux et dans toutes les réunions », pour que l'intercompréhension et l'intégration de chacun soient complètes.

C'est sous la forme d'une culture d'entreprise que la question du «langage commun » est principalement traitée dans les manuels de communication d'entreprise que nous avons étudiés, c'est-à-dire à la manière des précurseurs américains et français du management. Ainsi, dans leur manuel de communication, Philippe Détrie et Catherine Meslin-Broyez [2001:84] définissent le «langage commun » comme l'instauration d'un système de valeurs propres à l'entreprise. Cela signifie qu'il faudrait faire en sorte, par l'intermédiaire de la communication d'entreprise, d'organiser et de favoriser « les échanges d'information dans ses flux descendants, ascendants et transversaux », de diffuser " une information honnête et accessible aux différentes cibles internes de l'entreprise », de « faire de l'organisation et pour chacun un lieu où il fait bon travailler » et de favoriser « la promotion d'un même langage et d'un même référentiel pour que chacun puisse s'identifier à l'entreprise ou plus modestement s'intégrer dans son système de valeurs » [Détrie et Meslin-Broyez 2001 : 84]. Pour ce faire, on devrait mesurer « régulièrement les opinions et les attentes de ses différentes cibles » et évaluer « l'impact des actions qu'il [le communicant d'entreprise] a menées et leur cohérence avec la stratégie de

\footnotetext{
${ }^{4}$ Cette recommandation est présente dans $45 \%$ des vingt-deux manuels de communication d'entreprise que nous avons étudiés dans notre thèse (Mariscal 2015).
} 
l'entreprise ». Le communicant devra effectuer «ensuite les ajustements nécessaires » de manière à ce que les pratiques langagières et les comportements de chacune des partiesprenantes soient alignées sur les attentes de l'entreprise [Détrie et Meslin-Broyez 2001 : 85]. Le but est, au final, que les acteurs de l'entreprise acquièrent le langage propre à leur organisation, en d'autres termes qu'ils incarnent leur entreprise en l'utilisant quelle que soit la situation.

Cette forme d' «identité d'entreprise», pour reprendre l'expression utilisée par Nicole D'Almeida et Thierry Libaert [2010 : 13-14] qui pourrait s'apparenter à une forme d'identité nationale, de patriotisme, correspond à « un ensemble de caractéristiques indépendantes » donnant à l'entreprise, « à travers les individus, sa spécificité, sa stabilité et sa cohérence, et lui permet d'apparaître en propre et d'être reconnue ». En effet, l'«identité d'entreprise » par l'intermédiaire de la communication d'entreprise :

« traduit et organise la personnalité de la firme à travers une série cohérente de signes qui vont du plus visible (logo) au moins concret (opinions et comportements des employés) en passant par des étapes intermédiaires (telle la marque). L'identité d'une organisation est le système résultant de l'interaction de quatre types de facteurs : les facteurs politiques (histoire et personnalité des dirigeants, caractéristiques du pouvoir de tutelle), économiques (stratégie de produits et de développement), structurels (type d'organisation et de répartition du pouvoir, procédures et système de gestion retenu), et symboliques (rites, langages et symboles organisant l'équilibre interne du groupe social). Ce dernier point renvoie à un imaginaire organisationnel qui permet au salarié de se situer et de s'engager. Il s'agit là d'un des moteurs de la motivation et de l'implication de soi. » [D'Almeida et Libaert 2010 : 13]

Le but paraît donc clairement de réaliser une symbiose entre l'individu et l'entreprise, pour reprendre le terme utilisé par Nicole Aubert et Vincent de Gaulejac (1991 : 58).

Le langage est traité, à raison, comme un producteur de lien social, à l'instar d'autres éléments comme la construction et l'entretien de « rites (décorations, remise de médailles) » et de «symboles (taille des bureaux, tenue vestimentaire, organisation de sessions hors entreprise, etc.) » qui sont « constitutifs d'un processus d'identification par lequel l'individu se retrouve dans l'entreprise et y ajuste son comportement » [D'Almeida et Libaert $2010: 29$ ]. C'est à cette fin que la communication d'entreprise devrait créer « un langage commun qui permet à chacun d'orienter et d'adapter son comportement professionnel » [D'Almeida et Libaert $2010: 29$ ].

Nicole D'Almeida et Thierry Libaert [2010: 28-29] disent tirer la notion de «langage commun » des « champs ethnologique et anthropologique où elle désigne le ciment par lequel par lequel des personnes se sentent appartenir à la même société et existent comme une totalité organisée ». Dans le contexte de la communication, cela renvoie typiquement à la culture d'entreprise, c'est-à-dire à « une histoire, à des valeurs et à des pratiques que les communicants internes vont mettre en scène» [D’Almeida et Libaert 2010 :29]. Ainsi, si le «langage commun » est un «ciment» culturel, celui-ci est fabriqué par le management ou par la communication interne et n'est pas, à proprement parler, le produit d'un processus sociologique qui fait que les individus partagent les mêmes affects (Lordon $2015: 25,67$ ).

Le « langage commun » tel qu'il est inclus dans le concept d'identité d'entreprise, n'est pas non plus sans rappeler le storytelling (Salmon 2008), dont le but est de construire de toute pièce ou de réactualiser, en l'idéalisant, une histoire de l'entreprise et un système de valeurs par l'évocation «des grandes figures ou étapes de l'entreprise », ce passé faisant l'objet d'un embellissement qui «donne au groupe une origine, un sens et un ancrage » [D'Almeida et Libaert 2010 : 13]. Nous retrouvons ici le cas classique du système « managinaire » décrit par Nicole Aubert et Vincent de Gaulejac (1991), contraction de «management» et d' «imaginaire », que la communication d'entreprise cherche à construire en associant « langage commun » et culture commune, le premier étant le véhicule du second.

Ainsi, les théoriciens de la communication défendent l'idée que l'on peut intervenir sur le langage afin qu'il corresponde à un «imaginaire organisationnel » commun, permettant «au 
salarié de se situer et de s'engager », l'identité d'entreprise étant l'un «des moteurs de la motivation et de l'implication de soi » [D'Almeida et Libaert 2010 : 13]. Cette manière de vouloir relier et unifier le « corps social » dans l'entreprise [D'Almeida et Libaert $2010: 28$ ] pour permettre une communication horizontale, est donc à l'initiative du management et/ou des organes s'occupant de la communication interne, et résulte donc d'un mouvement vertical descendant.

2.3. Le « langage commun » comme code commun

Ce façonnement de l'identité des individus au travail devrait ainsi s'opérer par une intervention directe sur les pratiques langagières, en inventant ce que l'on pourrait appeler une «novlangue » façonnée à partir des intérêts propres à une entreprise, pour utiliser un terme issu de 1984 de George Orwell et repris, depuis, par nombre de commentateurs. Le «langage commun » fait donc partie d'un processus global, où chaque entreprise «s'invente un langage, un code déontologique et vestimentaire », qui sont autant d'éléments d'identification et de normalisation des comportements.

L'idée de «code commun » complète et précise la manière dont le «langage commun » est défini. En effet, le « langage commun » est érigé au rang de langue interne à l'entreprise, qui fait que chacun reste connecté, sans qu'il y ait d'échecs possibles dans les interactions. Ainsi, comme nous l'avons dit, le « langage commun » doit être valable en toutes circonstances et cela est d'autant plus vrai si on le considère comme un code commun.

Pour clarifier la manière dont ce code est défini, nous en avons étudié les différentes occurrences dans les manuels de communication d'entreprise analysés. Ramez Cayatte [2008 : 30] aborde la question du code commun selon l'angle de la standardisation, c'est-à-dire par la mise en place d'un « référentiel de communication », appelé à devenir la " "bible" de la communication ». Malgré cela, il s'agit surtout d'un document utile pour que chacune des parties-prenantes, dans le cadre d'un projet, possède une base commune pour travailler et soit en accord sur les principaux « arguments justifiant sa réalisation et un glossaire des mots-clés » [Cayatte 2008: 30]. Ainsi, nous pouvons supposer que ce type de «langage commun» ne concerne pas l'ensemble de l'entreprise, il est local, car il s'inscrit dans le cadre d'un projet particulier et ne couvre que des besoins pratiques et techniques valables à un moment donné.

Mais le «langage commun », défini comme un code commun, peut prendre un caractère plus englobant. Ainsi, pour André-A. Lafrance et François Lambotte [2008 : 33], il faudrait mettre en place un code de manière à ce que chacun puisse " "décoder" le langage (verbal ou écrit) utilisé » et ainsi être en mesure de «comprendre le manager». En effet, lors d'une « communication verticale, les employés ne pourront pas comprendre le manager s'ils ne sont pas initiés aux termes spécifiques du langage technique », par exemple de l'informatique, et le «langage devient une barrière à [la] communication de groupe car les employés sont susceptibles de ne pas comprendre les tenants et aboutissants du message » [Lafrance et Lambotte $2008: 34]^{5}$. Le salarié qui a des compétences moindres que son locuteur est donc un simple « décodeur » des messages émis par le management, et il est prêt à agir car il maîtrise le code avec lequel on s'adresse à lui. De cette manière, si le destinataire-décodeur «se met en situation de ne pas pouvoir être rejoint par le moyen/instrument utilisé par l'émetteur », il "déforme le contenu en lui appliquant, de façon plus ou moins consciente, un code inapproprié », et il devient alors un « interprète » qui « associe systématiquement les messages d'une certaine nature à un événement passé qui place ces messages dans un contexte défavorable dès leur émission» [Lafrance et Lambotte 2008: 52-53]. Dans ce cas, le destinataire-décodeur peut être défaillant.

\footnotetext{
${ }^{5}$ Les auteurs rapportent ici les propos de Loïc Delvosal.
} 
Un certain nombre de schémas de communication dans les manuels étudiés se présentent ainsi sous une forme minimale et horizontale, comme les deux exemples ci-dessous le montrent (figures 1 et 2) :

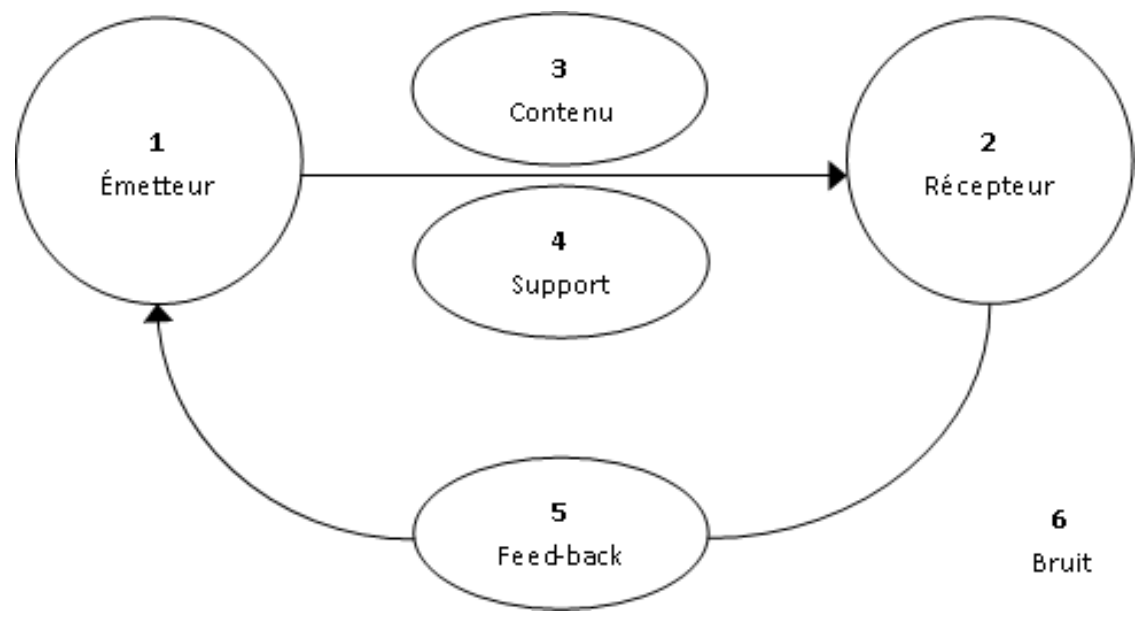

Figure 1 - Schéma de communication proposé par Pierre Guilbert [2007 : 19]

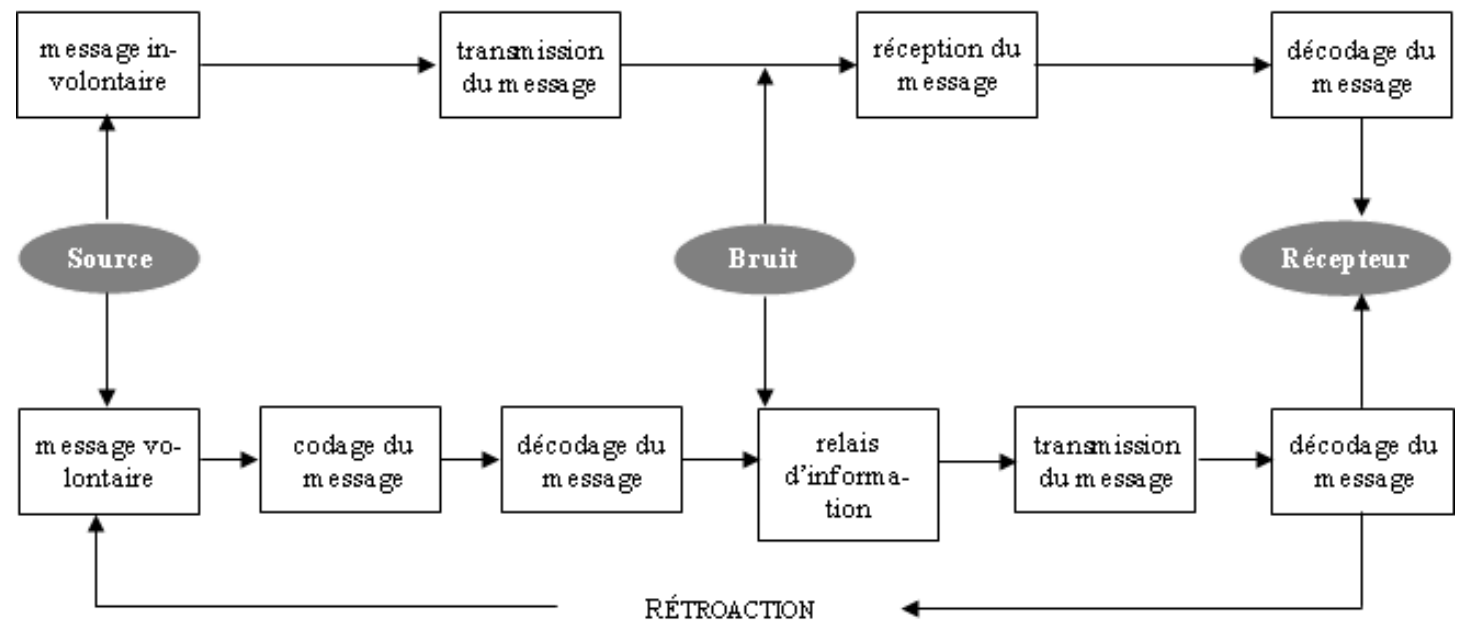

Figure 2 - « Un modèle théorique pour la communication interne » proposé par Jean-Marc Décaudin, Jacques Igalens et Stéphane Waller [2006 : 61]

Cette vision du langage est présente depuis plus de soixante ans dans les sciences de la communication, et rappelle celles des pionniers de la linguistique moderne, comme celle de Ferdinand de Saussure ([1916] 1972, figure 3) ou de Roman Jakobson (1963, figure 4). Le but est simplement de repérer les éléments fonctionnels essentiels permettant la transmission d'un message entre un locuteur et son destinataire, et ce type de modèle paraît, de cette manière, assez général pour être applicable en toutes circonstances. 


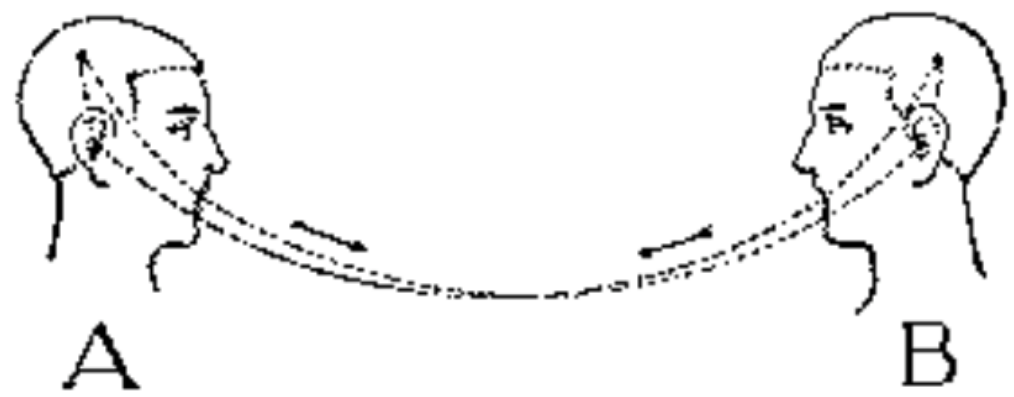

Figure 3 - Le circuit de la parole, d'après Louis-Ferdinand de Saussure ([1916] 1972 : 27)

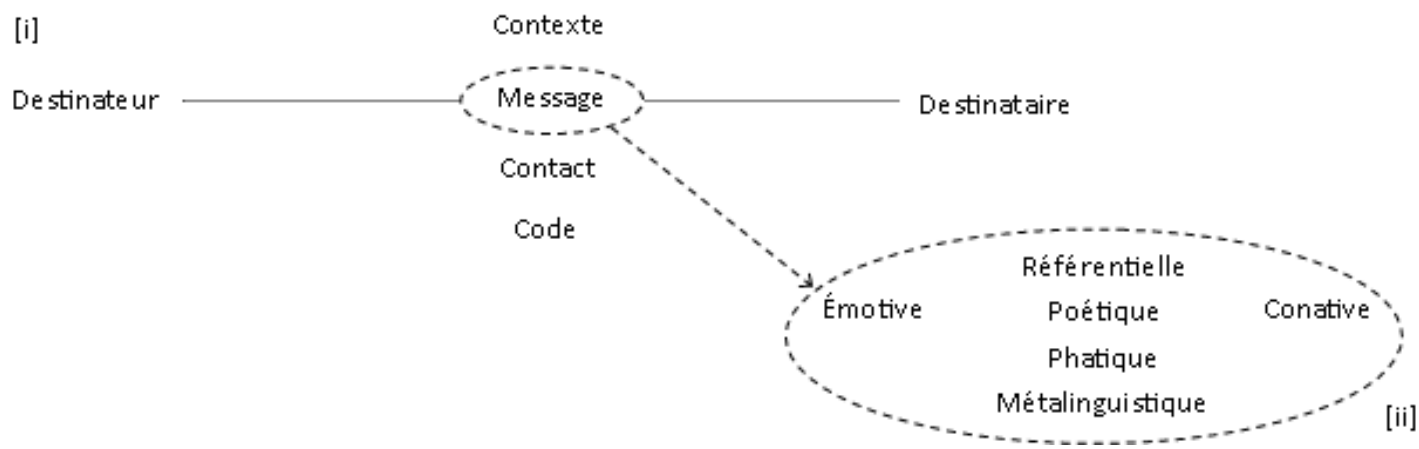

Figure 4 - Les facteurs « inaliénables » de la communication verbale [i] et les fonctions du langage qui leur sont associées [ii], d'après Roman Jakobson (1963:214, 220)

Mais, cette définition du langage a surtout subi l'influence de la « cybernétique » (Mattelart 2011) et, en particulier, de The Mathematical Theory of Communication proposée, à la fin des années 1940, par Claude Shannon et Warren Weaver ([1949] 1972) à la suite du mathématicien Norbert Wiener (1948) :

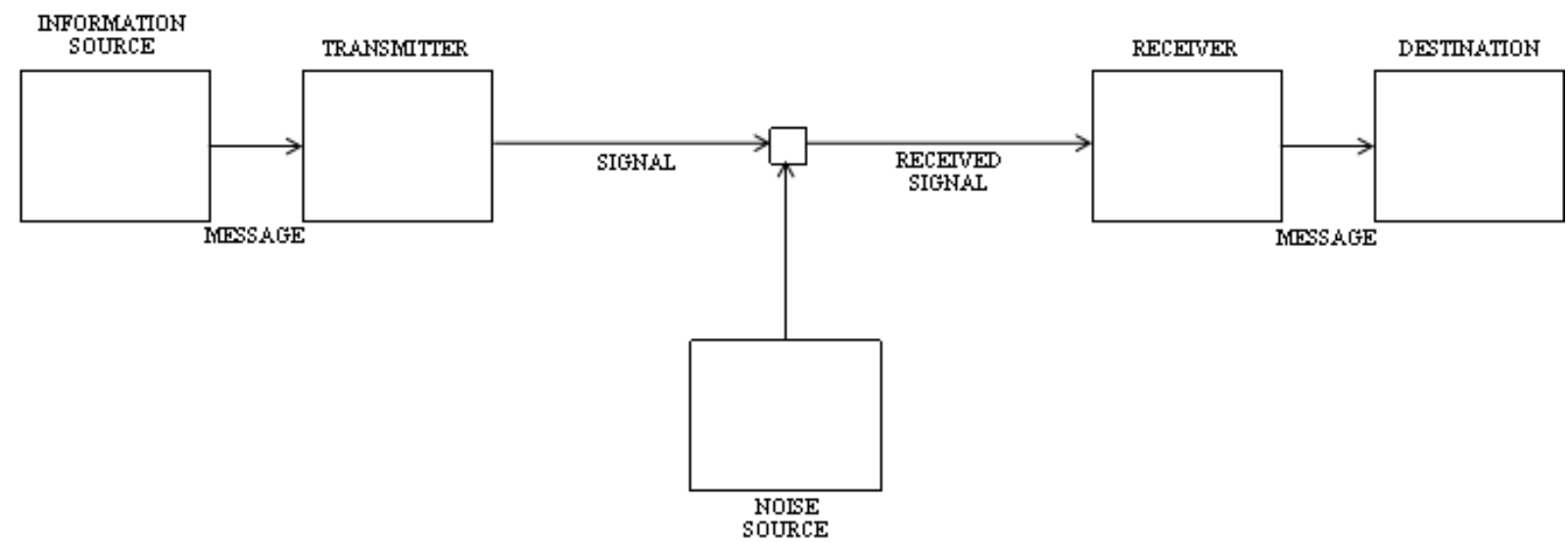

Figure 5 - Système de communication universel proposé par Claude Shannon et Warren Weaver ([1949] $1972: 7,34)$

Comme la figure 5 le montre, nous avons donc affaire, avec la cybernétique telle qu'elle est schématisée dans la Théorie mathématique, tout comme avec le langage tel qu'il est représenté dans les manuels, à une idée « très visuelle, très explicite, très "évidente" » du langage, où « le phénomène de la communication interindividuelle va de soi » (Winkin $2001: 39,53$ ), sans que 
l'on semble envisager la possibilité du raté, du malentendu et de l'incompréhension. C'est cet aspect en particulier qui va être approfondi dans la section suivante.

3. Le « langage commun » comme outil de dé-conflictualisation du rapport salarial

Il semblerait que l'objectif de l'instauration d'un « langage commun » dans une entreprise soit d'effacer toute asymétrie interactionnelle et sociale à travers la chaîne hiérarchique, en allant du haut vers le bas. Les problèmes posés par l'asymétrie dans les compétences langagières au travail ont été mis en évidence depuis un certain nombre d'années par la sociolinguistique et par la linguistique interactionniste (cf. Filliettaz 2007, Kerbrat-Orecchioni 1992 : 71, Vergely 2008). Mais, les théoriciens de la communication ne se positionnent pas par rapport à ce type d'études, puisque leur objectif est avant tout de créer une relation efficace, productive et mobilisatrice entre un point $\mathrm{A}$, le (top-)management et un point $\mathrm{B}$, les employés, mais aussi d'euphémiser les rapports symboliques de pouvoir, en particulier ceux passant par le langage (Bourdieu 2001), entre les différentes classes d'acteurs de l'entreprise.

Ainsi, dans les schémas reproduits ci-dessus, c'est la symétrie, l'horizontalité et la réciprocité qui dominent, même si cette réciprocité n'est figurée que par le «feedback» (figure 1) ou par la « rétroaction » (figure 2), c'est-à-dire par un simple retour sur expérience. Le fait de ne repérer que les éléments fonctionnels essentiels et de traiter le langage comme un code nous donne l'impression que les interactions langagières peuvent être, en effet, symétriques et qu'un unique modèle langagier peut être applicable en toutes circonstances. Cette vision relativement simple valide l'expertise des auteurs des manuels de communication d'entreprise ou, en tout cas, les éléments qui nous sont donnés ne nous permettent pas de remettre en question sa logique interne, sans apporter d'éléments extérieurs.

Nous remarquons également qu'un certain flou existe quant au véritable prescripteur du « sens » et des valeurs communes que le « langage commun » devrait véhiculer. Par exemple, lorsque Jean-Pierre Béal, Franck Frommer et Pierre-André Lestocart [2003 : 11] nous indiquent que le rôle du communicant est de « produire du sens et expliquer », on ne sait pas du sens de quoi il s'agit, ni qui le construit. La communication d'entreprise est ainsi présentée comme étant sans sujet, indéfinie et intransitive, le message des manuels se réduisant à l'injonction suivante : il « faut de l'échange, de l'argumentation, de la pédagogie, bref de la parole (voire de l'émotion) pour convaincre, être convaincu et vouloir changer de comportement » [Béal, Frommer et Lestocart $2003: 28]$.

La vision du langage défendue dans les manuels de communication nous paraît correspondre à l'utopie que Bernard Floris (1996 : 226) appelle une «idéologie managériale consensuelle et intégrative ». Cette tendance à idéaliser les pratiques langagières en prétendant offrir aux entreprises la possibilité d'une communication censément horizontale et d'une intercompréhension parfaite contribue à effacer le caractère éminemment coercitif de l'idée de « langage commun », qui s'oppose au projet de libération de la parole au travail. Qui plus est, l'idée que l'on puisse fabriquer de toute pièce un « langage commun », nous laisse à penser que l'on veut donner aux faits sociaux et langagiers « une prévisibilité relative », voire totale (Coutrot 1998 : 169), atteindre une maîtrise dépassant toutes contraintes socioculturelles et contextuelles.

Plus largement, comme nous l'avons montré ailleurs (Mariscal 2016), les auteurs des manuels de communication d'entreprise comme ceux de management, éludent la question de la verticalité, c'est-à-dire du conflit inhérent au rapport salarial. Ainsi, l'utopie d'une communication horizontale correspond à la vision néolibérale d'une société a-institutionnelle, d'une possible existence autonome et détachée des rapports de pouvoir (Lordon 2015 : 25, 56). Mais, comme Frédéric Lordon (2015 : 217) le montre, l'horizontalité est aussi une puissance normalisatrice et la communication, telle qu'elle est présentée dans les manuels n'est, en dernière analyse, qu'une « pétition de principe donnant le problème pour résolu d'avance ». 
D'ailleurs, le philosophe montre que, si une communauté peut être rendue horizontale, cette horizontalité est toujours provisoire, car elle est prise dans une perpétuelle instabilité passionnelle (Lordon 2015 : 318).

La communication d'entreprise se veut également résolument interculturelle, dépasser les barrières idéologiques, les clichés et les cultures nationales, conformément à l'esprit du management depuis le début des années 1980 (Cusset 2008, Mariscal 2015). Ainsi, paradoxalement, malgré un appel à la normalisation et à la standardisation, il faudrait comprendre « la spécificité des cultures dans lesquelles intervient l'entreprise », et « rechercher ce qui peut être accepté par elles et de miser sur les complémentarités » [D'Almeida et Libaert 2010 : 122]. Il apparaît donc que, dans le cadre de la mondialisation, on voudrait donner l'illusion aux acteurs d'appartenir à un collectif, à un « village planétaire » (Breton 1992), luimême fondé sur les idées de «libre-échange » (Mattelart 2011), d' «autonomie », de « responsabilité » dans une communauté humaine unifiée, sans classes sociales, où l'intercompréhension est une évidence (Clot 2008 : 12-13, 16, 21). C'est ce qui permettrait, toujours conformément à l'idéologie néolibérale, d'éloigner la «tentation communautaire » tout en créant une communauté de travail partageant strictement la même culture [D'Almeida et Libaert 2010 : 30]. Finalement, le discours des manuels de communication, semble s'inscrire dans le cadre d'un néolibéralisme cherchant à résoudre, tout du moins de manière performative, toutes contradictions. Ce discours rend, ainsi, l'utopie de l'horizontalité parfaitement réalisable en mettant en scène une société où tous les «sujets libres » pourraient nouer librement des rapports (Lordon 2015 : 69), où les interactions langagières seraient symétriques.

Nous relions cela à une interprétation particulière de la démocratie, que l'on présente comme étant le «fruit d'expériences empiriques et non idéologiques », comme une "synthèse historique globale » où toute discontinuité, comme le conflit, n'est acceptable que si elle ne remet pas en cause les fondements du système (Benasayag et del Rey 2007 : 16-17, Mariscal 2016). Selon Miguel Benasayag et Angélique del Rey (2007 : 17), ce caractère absolu « est masqué par la revendication assumée de la controverse, des opinions divergentes comme le respect de l'antagonisme des intérêts. À ceci près que ces controverses et divergences n'ont le droit d'exister que dans le cadre d'une normalisation intérieure au système ».

Il ne s'agirait donc pas, à travers la culture d'entreprise, de manipuler les acteurs, mais de les faire consentir volontairement à marcher ensemble en les intégrant, en particulier par le langage, à un même "système de valeurs » [Détrie et Meslin-Broyez 2001 : 84]. Il n'en est pas moins que «langage commun » continue de rimer avec vision commune et plus difficilement avec interculturalité ou pluralisme, car il s'agit bien de créer, pour reprendre l'expression de Thomas Peters et Robert H. Waterman (1983: 262), une « grande famille », par l'instauration du consensus et par l'universalisation des intérêts particuliers, dont Bourdieu (1977: 408) fait la définition de l'idéologie elle-même.

Le management s'en trouve désincarné, on ne connaît que les fins du dispositif, qui sont de fluidifier les relations entre le haut et le bas de la hiérarchie de manière à rendre l'entreprise efficace, par une intervention directe sur les éléments de langage qui y circulent, sans nous dire quels sont, exactement, les moyens envisagés. Nous savons seulement, par l'intermédiaire du manuel de Christine Donjean [2007 : 100], qu'il faudrait mettre en place de véritables «plans de formation » pouvant s'étendre sur plusieurs mois, voire sur plusieurs années. Le but est de s'assurer que tous les acteurs comprennent et intègrent les «attitudes » et les «conceptsclés requis par l'organisation », « en d'autres termes que l'on parle le même langage » [Donjean 2007 : 100]. Le «langage commun » demanderait ainsi un véritable investissement de la part de l'entreprise pour le mettre en place, et de la part de l'ensemble des acteurs pour l'acquérir. 


\section{Conclusion}

Nous avons vu, tout au long de cet article, que le langage au travail avait évolué selon une trajectoire spécifique depuis les années 1980, en passant du statut de quasi-interdit à une volonté, de la part du management, de l'intégrer parfaitement au fonctionnement de l'organisation. Il semble que les théoriciens du management aient alors pris conscience de ses enjeux stratégiques, notamment en termes de participation, de mobilisation mais aussi de maîtrise possible du langage au travail ainsi libéré. Il s'agit, désormais, de connecter les individus, de fluidifier les relations au travail, avant tout du haut vers le bas de la hiérarchie. Pour ce faire, la communication d'entreprise devrait se donner pour but de résoudre des contraintes socioculturelles. Ainsi, la libération du langage au travail et l'horizontalisation de l'entreprise masquent d'autres modes de contrôle, de normalisation, non moins coercitifs que ceux que l'on a connus jusqu'ici, mais aussi le déni des rapports conflictuels et passionnels inhérents à toute relation sociale, en particulier au travail. De cette manière, le «langage commun » correspond à une utopie basée sur une homogénéisation linguistique et culturelle particulièrement stricte, notamment lorsqu'il est défini comme un code commun.

Derrière un discours mettant en scène la démocratisation ainsi que la fin du paternalisme et du taylorisme, nous avons un discours pratique et pragmatique, qui suggère le remplacement de la culture des individus par une culture entrepreneuriale endémique. Paradoxalement, il faudrait éviter tout communautarisme et toute idéologie, conformément à la pensée néolibérale. Enfin, nous avons vu que les moyens préconisés pour imposer le « langage commun » restaient dans le flou. On n'en connaît que les fins dont les aspects utopiques peinent à masquer un objectif classique qui est le contrôle intégral, par le management, du système de production.

En effet, le problème central posé par la notion de «langage commun » est de prétendre qu'une homogénéisation des pratiques langagières au travail est possible et nécessaire, alors même que les rapports socioculturels témoignent, bien au contraire, d'une hétérogénéité fondamentale. D'une part, il semble que le management cherche ainsi à résoudre les contradictions inhérentes aux rapports sociaux de production, mais aussi à euphémiser un rapport salarial intrinsèquement conflictuel. D'autre part, comme Luc Boltanski et Ève Chiapello (1999) l'ont montré, le management tel qu'il a cherché à se réinventer au début des années 1980, vient combler le vide laissé par un discours syndical construit comme une base identitaire sur laquelle toute culture salariale devait venir reposer. Comme Bernard Gardin (2005 : 279, 281) l'a analysé, le discours syndical des années 1970 faisait comme «si les travailleurs devaient abandonner leurs caractéristiques linguistiques » pour adhérer à des formes standardisées plus adaptées aux luttes sociales contemporaines. Mais, le discours managérial, au lieu de tendre vers l'émancipation de l'individu, a débouché sur la prescription d'une forme de solidarité «mécanique », «passive », «imposée par les conditions de production» par le biais des pratiques langagières. Ainsi, la capacité d'expression des travailleurs qui fut le vœu, concomitamment, de la loi Auroux de 1982 et du nouveau management, en sort détériorée par le «langage commun ». Cet outil est donc un exemple de lutte «contre la privation de parole» et donc «contre l'aliénation » aboutissant, paradoxalement, à une nouvelle « conquête » de la parole au travail (Gardin 2005 : 284). Il apparaît que les schémas de communication occupent eux-aussi une fonction essentielle de réduction de la compréhension du langage au travail en lui imposant une « raison graphique » bidimensionnelle et figée (Goody 1979 cité par Gardin 2005 : 335, Foucault 1966).

La libération effective de la parole au travail, telle qu'elle est présentée dans les manuels de communication, est donc toute relative. De l'aveu même des auteurs des manuels, cette parole « libérée » par un système participatif se trouve remodelée dans le meilleur des cas à une fin « pédagogique » et de cohésion, en garantissant « une cohérence de la parole de l'entreprise, mais en la rendant moins monolithique, pour orienter le salarié dans le flux de connaissances et d'informations qui lui est proposé » [Béal, Frommer et Lestocart 2003 : 11]. 
L'autre aspect important que nous voyons apparaître, en filigrane, avec le «langage commun », est la réduction de la problématique des interactions langagières à l'utilisation des « mots les plus simples » qui, certes, « ne mettent pas en valeur la richesse de votre vocabulaire ni votre érudition, mais [qui] vous donnent l'assurance d'être compris du plus grand nombre » [Sananès 2011 : 133]. Nous constatons donc que le souci de la communication d'entreprise, telle qu'elle apparaît dans les manuels étudiés, est de viser toujours plus d'efficacité et donc de rentabilité, par la réduction à une vision simple, voire simpliste, des rapports sociaux. Cet esprit correspond bien à celui du management initié au début des années 1980, pour qui la forme précède toujours le fond ${ }^{6}$, où l'essentiel est toujours d'être compris. Cela démontre la concentration de la communication d'entreprise sur le message qu'elle veut faire passer, sur l'image que l'entreprise veut donner d'elle-même en interne ou à l'extérieur car, comme Pierre Guilbert [2007 : 55] l'affirme dans son manuel « l'important n'est pas ce qui est dit ; l'important est ce qui est compris », et le message doit passer, quoi qu'il arrive.

Pour conclure, la communication devrait ainsi opérer un travail sur les salariés pour qu'ils soient accessibles au (top-)management et qu'ils travaillent de manière efficace selon ses recommandations et la ligne qu'il a tracée. Le but est de rendre le salariat " communicant", mais selon des règles spécifiques imposées par le haut, ce qui nous permet de douter de l'horizontalisation effective de la communication telle qu'elle est promue à travers le « langage commun », le caractère descendant demeurant au centre de cette vision de la communication. Ainsi, l'organisation de l'entreprise reste scindée, d'une certaine manière, entre décideurs et exécutants, comme ce fut le cas dans le cadre du taylorisme, ce que Danièle Linhart (2015) a montré, par ailleurs, dans sa comparaison entre le management contemporain et The Principles of Scientific Management de Frederick W. Taylor ([1911] 2003). Mais le «langage commun » a pour spécificité d'être également une entreprise de persuasion, dans le sens où elle implique d'agir toujours plus profondément sur la subjectivité des acteurs (Mariscal 2015).

Références bibliographiques :

Albert M. (1982), Le pari français : le nouveau plein-emploi, Paris, Seuil.

Albert M. (1983), Un pari pour l'Europe : vers le redressement de l'économie européenne dans les années 80, Paris, Seuil.

Archier G. (1981), Le soleil se lève à l'ouest, Paris, Sofedir.

Aubert N. et de Gaulejac V. (1991), Le coût de l'excellence, Paris, Seuil.

Béal J.-P., Frommer Fr. et Lestocart P.-A. (2003), Entre management et marketing: la communication interne, Paris, Démos.

Benasayag M. et del Rey A. (2007), Éloge du conflit, Paris, La Découverte.

Boltanski L. et Chiapello È. (1999), Le nouvel esprit du capitalisme, Paris, Gallimard.

Bourdieu P. (1977), «Sur le pouvoir symbolique», Annales. Economie, Sociétés, Civilisations 32, p. 405-411.

Bourdieu P. (2001), Langage et pouvoir symbolique, Paris, Seuil.

Boutet J. (2008), La vie verbale au travail. Des manufactures aux centres d'appels, Toulouse, Octarès.

Breton P. (1992), L'utopie de la communication : le mythe du «village planétaire », Paris, La Découverte.

Castells M. (1999), L'ère de l'information, tome 1 : la société en réseaux, Paris, Fayard.

Cayatte R. (2008), Communiquer et convaincre dans un projet, Paris, Eyrolles.

Clot Y. (2008), Le travail sans l'homme? Pour une psychologie des milieux de travail et de vie, Paris, La Découverte.

\footnotetext{
${ }^{6}$ «Les entreprises qui sont tournées vers le personnel possèdent des langages qui se ressemblent. Sous bien des aspects, la forme précède le fond » (Peters et Waterman $1982: 261$ ).
} 
Coutrot T. (1998), L'entreprise néo-libérale, nouvelle utopie capitaliste, Paris, La Découverte. Cusset F. (2008), La décennie. Le grand cauchemar des années 1980, Paris, La Découverte.

D'Almeida N. et Libaert T. (2010), La communication interne des entreprises, Paris, Dunod.

Décaudin J.-M., Igalens J. et Waller S. (2006), La communication interne: stratégies et techniques, Paris, Dunod.

Détrie P. et Meslin-Broyez C. (2001), La communication interne au service du management, Paris, Liaisons.

Donjean C. (2007), La communication interne, Liège, Edipro.

Filliettaz L. (2007), «Interactions professionnelles et rythme de l'action. Contribution à une approche praxéologique du temps », dans Chabrol C. et Olry-Louis I. (éd.), Interactions communicatives et psychologie, Paris, Presses de la Sorbonne Nouvelle, p. 179-192.

Floris B. (1996), La communication managériale: la modernisation symbolique des entreprises, Grenoble, Presses Universitaires de Grenoble.

Foucault M. (1966), Les mots et les choses, Paris, Gallimard.

Gardin B. (2005), Paroles d'ouvrières et d'ouvriers, Limoges, Lambert-Lucas.

Goody J. (1979), La raison graphique. La domestication de la pensée sauvage, Paris, Éditions de Minuit.

Guilbert P. (2007), Le B.A.-BA de la communication à l'usage de toute personne qui souhaite séduire, informer, convaincre : de Gutenberg à l'Homo Connectus, Liège, Edipro.

Jakobson R. (1963), Essais de linguistique générale, tome 1, Paris, Les éditions de Minuit.

Kerbrat-Orechioni C. (1992), Les interactions verbales, tome 2, Paris, Armand Colin.

Lafrance A.-A. et Lambotte F. (2008), Arrêtez de communiquer, vous en faites trop! Pour un développement durable des réseaux de communication dans l'entreprise, Montréal, Éditions Nouvelles.

Le Goff J. (1989), Du silence à la parole : droit du travail, société, État (1830-1989), Quimper, Calligrammes.

Linhart D. (2015), La comédie humaine du travail : de la déshumanisation taylorienne à la surhumanisation managériale, Toulouse, Érès.

Lordon F. (2010), Capitalisme, désir et servitude, Paris, La Fabrique.

Lordon F. (2015), Imperium : théorie des ensembles souverains, Paris, La Fabrique.

Mariscal V. (2015), "Soyez sans crainte». Normalisation langagière et comportementale au travail en contexte capitaliste néolibéral : une analyse critique de manuels de management et de communication d'entreprise, Université catholique de Louvain, Louvain-la-Neuve.

Mariscal, V. (2016). «Entreprise du $3^{\text {e }}$ type et "dé-passionnalisation" de la vie organisationnelle: quand le désaccord se dissout dans une culture du consensus », dans Wagener A., Ravat J. et Nicolas L. (éd.), La valeur du désaccord, Paris, CNRS éditions.

Mattelart A. (2011), L'invention de la communication, Paris, La Découverte.

Maury R. (1986), Marianne à l'école japonaise : la réussite du Japon au service des Français, Paris, Plon.

Maury R. (1990), Les patrons japonais parlent, Paris, Seuil.

Morel P. (2009), La communication d'entreprise, Paris, Vuibert.

Nahon S. et Taskin L. (2009), e-GRH: Enjeux et perspectives : informer, collaborer et impliquer, Liège, Edipro.

Orwell G. ([1949] 1972), 1984, Paris, Gallimard.

Peters T. et Waterman R. H. (1983), Le prix de l'excellence. Les secrets des meilleures entreprises, Paris, InterÉditions.

Salmon C. (2008), Storytelling : la machine à fabriquer des histoires et à formater les esprits, Paris, La Découverte.

Sananès B. (2011), La communication efficace : acquérir maîtrise et confiance en soi dans ses rapports avec les autres, Paris, Dunod. 
Saussure F. de (1916), Cours de linguistique générale, Paris, Payot.

Sérieyx H. (1982), Mobiliser l'intelligence de l'entreprise. Cercles de qualité et Cercles de pilotage, Paris, EME.

Shannon C. et Weaver, W. ([1949] 1972), The Mathematical Theory of Communication, Urbana, Chicago, London, University of Illinois Press.

Taylor F. W. ([1911] 2003), The Principles of Scientific Management, New York, Dover Publications Inc.

Vergely P. (2008), «L'expression du dysfonctionnement technique dans les interactions orales de travail », Langage et Société 125, p. 35-54.

Wiener N. (1948), Cybernetics or Control and communication in the animal and the machine, Paris, Hermann.

Winkin Y. (2001), Anthropologie de la communication : de la théorie au terrain, Paris, Seuil. 